\title{
Configurações
}

Revista de sociologia

16 | 2015

Violência e Criminalidade

\section{Fatores de risco de violência contra as pessoas idosas: consensos e controvérsias em estudos de prevalência}

Risk factors for violence against older adults: consensus and controversies in prevalence studies

Les facteurs de risque de violence contre les personnes âgées: les consensus et les

différends dans les études de la prévalence

Ana Paula Gil, Ana João Santos, Rita Nicolau e César Santos

\section{OpenEdition}

\section{Journals}

Edição electrónica

URL: http://journals.openedition.org/configuracoes/2852

DOI: $10.4000 /$ configuracoes.2852

ISSN: 2182-7419

Editora

Centro de Investigação em Ciências Sociais

Edição impressa

Data de publição: 18 Dezembro 2015

Paginação: 75-95

ISBN: 1646-5075

ISSN: 1646-5075

\section{Refêrencia eletrónica}

Ana Paula Gil, Ana João Santos, Rita Nicolau e César Santos, «Fatores de risco de violência contra as pessoas idosas: consensos e controvérsias em estudos de prevalência », Configurações [Online],

16 | 2015, posto online no dia 29 dezembro 2015, consultado o 19 abril 2019. URL : http://

journals.openedition.org/configuracoes/2852 ; DOI : 10.4000/configuracoes.2852 
Gil, Ana Paula; Santos, Ana João; Nicolau, Rita; Santos, César - Fatores de risco de violência contra as pessoas idosas: consensos e controvérsias em estudos de prevalência.

Configurações, vol. 16, 2015, pp. 75-95

\section{Fatores de risco de violência contra as pessoas idosas: consensos e controvérsias em estudos de prevalência}

ANA PAULA GIL*

Instituto Nacional de Saúde Dr. Ricardo Jorge

ANA JOÃO SANTOS**

Instituto Nacional de Saúde Dr. Ricardo Jorge

RITA NICOLAU**

Direção-Geral do Território

CÉSAR SANTOS****

Instituto Nacional de Medicina Legal e Ciências Forenses

\section{Resumo}

O presente artigo discute os fatores de risco associados às condições de ocorrência da violência contra as pessoas idosas em contexto familiar, abordando os modelos teóricos que fundamentam a sua génese. Com base numa revisão da literatura, destacam-se os fatores de risco identificados por estudos de base populacional desenvolvidos nas três últimas décadas, especialmente na Europa e nos países anglo-saxónicos. O restrito número de casos de violência diferenciados a partir de amostras de base populacional tem repercussões na determinação dos fatores de risco e nas metodologias adotadas para a sua estimação. Nos estudos analisados os potenciais fatores de risco descritos resultam na sua maioria da análise de frequências aplicada às características de vítimas e agressores e aos aspetos contextuais e/ou socioculturais. Os modelos teórico-conceptuais subjacentes raramente são abordados.

Palavras-chave: envelhecimento, violência, pessoas idosas, família, fatores de risco.

\footnotetext{
* Doutorada, investigadora no Departamento de Epidemiologia do Instituto Nacional de Saúde Doutor Ricardo Jorge (INSA) e docente no Departamento de Sociologia da FCSH-UNL. E-mail: ana.gil@insa. min-saude.pt; anapgil@fcsh.unl.pt.

** Mestre, investigadora no Departamento de Epidemiologia do Instituto Nacional de Saúde Doutor Ricardo Jorge (INSA). E-mail: ana.carvalho@insa.min-saude.pt.

*** Doutorada, investigadora na Direção-Geral do Território. E-mail: rnicolau@dgterritorio.pt.

**** Mestre em Medicina, Médico Forense no Instituto Nacional de Medicina Legal e Ciências Forenses, IP. E-mail: cesar.santos@dcinml.mj.pt.
} 


\section{Abstract \\ Risk factors for violence against older adults: consensus and controversies in prevalence studies}

This paper presents a literature review on risk factors associated with violence against community-dwelling older adults. The approach also considers the theoretical models that underlying the identification of risk factors. The literature review highlights risk factors identified by population-based studies developed in the past three decades, especially in Europe and Anglo-Saxon countries. The restricted number of violence cases obtained by population-based studies impacts the identification of risk factors and the methodologies adopted for their estimation. The analysis of the potential risk factors described results, for the most part, of frequency analysis applied to the victims' and aggressors' characteristics, as well as contextual and socio-cultural aspects. The theoretical and conceptual models are rarely addressed.

Keywords: aging, violence, older adults, family, risk factors.

\section{Résumé \\ Les facteurs de risque de violence contre les personnes âgées : \\ les consensus et les différends dans les études de la prévalence}

Cet article aborde les facteurs de risque associes aux conditions d'apparition de la violence à l'égard des personnes âgées dans un contexte familial, en abordant les modèles théoriques qui soutiennent sa genèse. À l'aide de la littérature, il met en évidence les facteurs de risque identifiés par les études populationnelles développées au cours des trois dernières décennies, notamment en Europe et dans les pays anglo-saxons. Le nombre limité de différents cas de violence, compte tenu des échantillons représentatifs de la population, a une répercussion sur la détermination des facteurs de risque et sur les méthodologies adoptées pour leur estimation. Suivant les études analysées, les facteurs de risque potentiels adviennent principalement de l'analyse de la fréquence appliquée aux caractéristiques des victimes, des agresseurs et aux aspects contextuels et/ou socioculturelles. Les modèles théoriques et conceptuels sous-jacents sont rarement pris en compte.

Mots-clés: vieillissement, violence, personnes âgées, famille, facteurs de risque.

\section{Introdução}

A violência contra as pessoas idosas não constitui um problema recente, mas adquire hoje maior visibilidade com o aumento absoluto e relativo do número de pessoas idosas e da sua longevidade. A designada crise da família, relacionada com a perda de valores ditos tradicionais e práticas sociais no seu seio, é um dos argumentos invocados para a construção social do abandono das pessoas idosas pelos seus familiares. No entanto, nas últimas décadas, inúmeros trabalhos científicos vieram realçar a importância da família nas trocas intergeracionais (Coenen-Huther, Kellerhals e Allmen, 1994; Fernandes, 1997; Gil, 
2010). Se a família é representada como um espaço de afetos e reciprocidades, pode simultaneamente constituir um lugar de omissões e de violência.

É o olhar sobre a violência em contexto familiar que aqui se aborda. Os primeiros trabalhos desenvolvidos para explicar este fenómeno assentavam em explicações individuais, inserindo-se nos modelos psicológico e sociológico, este último assente em fatores sociossituacionais (Burnight e Mosqueda, 2011). O stress de quem cuida, o isolamento social, as condições socioeconómicas, a incapacidade física e mental da pessoa idosa, os problemas de saúde mental e os problemas aditivos dos agressores são algumas das causas apontadas (Tortosa, 2004).

O artigo revê criticamente os modelos teóricos sobre a violência contra as pessoas idosas, desenvolvidos na última década e analisa os fatores de risco (individuais, contextuais e estruturais) associados às condições da sua ocorrência. Destacam-se os fatores de risco identificados por estudos de prevalência, relativamente aos quais se explicitam os métodos-estatísticos utilizados.

\section{Método}

A identificação de estudos de interesse fundamentou-se numa revisão crítica da literatura sobre os fatores de risco em estudos de prevalência de base populacional sobre a violência contra as pessoas idosas, que foi publicada nas três últimas décadas. A Biblioteca do Conhecimento Online (B-On) foi privilegiada como principal meio de pesquisa desenvolvida com recurso a palavras-chave da temática em inglês e em português: violência em contexto familiar, pessoas idosas, fatores de risco, estudos de prevalência de base populacional.

Dos estudos publicados no período 1990-2012, excluíram-se os meramente descritivos, os qualitativos, os quantitativos baseados em amostras de conveniência e os estudos sobre violência institucional. Os critérios de seleção utilizados foram: utilização de amostras representativas das populações visadas, dando preferência a estudos de âmbito nacional; documentação dos métodos, instrumentos de recolha e da análise estatística dos dados.

Os critérios descritos conduziram à seleção de nove estudos (Quadro 1) representativos de sete países europeus e anglo-saxónicos, em que o objetivo principal foi a estimação da prevalência do fenómeno da violência contra pessoas idosas, sendo a identificação dos principais fatores de risco um objetivo secundário viabilizado pela existência de amostras representativas da população em geral.

Nos estudos epidemiológicos propõe-se a designação "definição de caso", elemento central que tem como objetivo estimar a prevalência, terminologia com pouca tradição na área das ciências sociais e humanas. A taxa de prevalência (TP) corresponde à razão entre o número total de casos (novos e antigos) de um determinado evento (por exemplo, doença, comportamento, ou 
qualquer outra característica individual ou de relevo para a saúde do indivíduo ou da população) ocorridos numa região ou país, num determinado período de tempo, relativamente à população total dessa região ou país, no mesmo período. Geralmente é expressa por 100 habitantes.

Contrariamente ao objeto da Epidemiologia, não estamos perante uma doença, mas sim uma ocorrência de vitimação com frequência variável e um dano que pode ser de natureza física, psicológica, financeira, sexual e negligente.

\section{Resultados e discussão}

\subsection{Modelos teóricos explicativos da violência contra as pessoas idosas}

A primeira dificuldade no estudo da violência diz respeito às definições de violência, abuso e maus-tratos. A Organização Mundial de Saúde (OMS) adota a definição da violência contra as pessoas idosas da Action on Elder Abuse (1995): "um ato único ou repetido, ou a falta de uma ação apropriada, que ocorre no âmbito de qualquer relacionamento onde haja uma expectativa de confiança, que cause mal ou aflição a uma pessoa mais velha" (OMS/INPEA, 2002: 3).

Três das dimensões centrais na definição adotada apresentam especificidades ao nível da sua operacionalização:

- Adulto idoso - a faixa etária adotada não é unânime. Os estudos de prevalência incluem, maioritariamente, indivíduos com 60 e mais anos ou com 65 e mais anos (Grande et al., 2000; Lowenstein, Eisikovits e Band-WWinterstein, 2009; Marmolejo, 2008; Naughton et al., 2012; Pillemer e Finkelhor, 1988; Podnieks, 1993).

- Relação interpessoal de confiança - pode incluir membros da família, amigos, vizinhos ou mesmo cuidadores formais (profissionais remunerados). Alguns autores (Naughton et al., 2012; O'Keefe et al., 2007) só contemplam agressores conhecidos ou do círculo de relações da vítima.

- Conduta ou ato, variável de acordo com o tipo (física, psicológica, financeira, sexual e negligência) - provoca um efeito, traduzido num dano físico e/ou mental.

Apesar de a definição adotada pela OMS ter alcançado grande popularidade, esta tem sido operacionalizada diferenciadamente entre estudos nas dimensões da análise, teorias explicativas e fatores de risco.

As abordagens teóricas explicativas são diversas, dando maior ou menor enfoque a fatores psicológicos e sociais. Se, por um lado, grande parte dos modelos provém dos maus-tratos infantis e da violência conjugal (Burnight 
e Mosqueda, 2011), por outro lado, a multiplicidade de teorias será também influenciada pela heterogeneidade de fatores de risco apontados pela investigação (Schiamberg e Gans, 1999).

De facto, algumas das teorias centram-se em fatores de risco identificados na literatura, podendo ser agrupadas em quatro abordagens: interpessoal, intrapessoal, multissistémica e sociocultural (Burnight e Mosqueda, 2011).

$\mathrm{Na}$ abordagem interpessoal três teorias focam a relação entre vítima e agressor: stress do cuidador, troca social e díade de discórdia. A teoria do stress do cuidador preconiza a origem da violência numa sobrecarga do cuidador, incapaz de responder às necessidades da pessoa cuidada (Burnight e Mosqueda, 2011). Os cuidados familiares são concebidos como uma experiência negativa (Zarit, Reever e Bach-Peterson, 1980) e como espaço propício à emergência de violência. Seria um fenómeno situacional que ocorreria pelo isolamento social e condições socioeconómicas adversas, associadas ao longo período de prestação de cuidados (Lachs et al., 1997; Paveza et al., 1992). Apesar de as práticas de cuidados poderem ser geradoras de situações de esgotamento físico e mental, estabelecer uma relação unívoca entre a violência/negligência e o stress do cuidador parece-nos uma arriscada análise de causalidade (Gil, 2010).

Os estudos de prevalência indicam que só uma minoria de pessoas idosas que necessita de algum tipo de cuidados é de facto vítima (Pillemer, 2005). Nos estudos revistos por Pillemer (2005) não houve evidência de diferenças significativas no nível de stress entre cuidadores abusivos e não abusivos.

A teoria da troca social explica as interações entre os indivíduos como um processo de trocas negociadas, onde circulam apoios recíprocos (domésticos, materiais, financeiros, ajudas na habitação, serviços), que são indissociáveis das normas de obrigação e de reciprocidade familiar (Bengston, 2001) e implicam relações ambivalentes de desigualdade, poder e dependência (Luescher e Pillemer, 1998).

O envelhecimento e a situação de dependência e/ou o baixo estatuto social percecionado implicariam uma alteração de poder na relação e reciprocidade da contribuição de cada elemento (Burnight e Mosqueda, 2011). Um cuidador poderá sentir maior poder, mas, simultaneamente, menor recompensa na relação, emergindo a relação abusiva. Uma das críticas dirigidas à teoria da troca social reside na exclusão dos determinantes macrossociais e na posição social desigual e de dependência atribuída à pessoa idosa (Sever, 2009), como se a dependência marcasse a dissolução da relação familiar e o fim da reciprocidade social (Lavoie, 2000). Além disso, alguns estudos apontam para a dependência (emocional e financeira) do cuidador face à vítima enquanto fator de risco da violência (Pillemer e Finkelhor, 1988).

A teoria da díade da discórdia, também denominada de "violência bidirecional", assenta no pressuposto que a reciprocidade da violência faz dos próprios cuidadores, também vítimas, sendo a violência, por isso, bilateral e resultante 
da interação social. Paveza e colaboradores (1992) demonstram que as famílias com doentes de Alzheimer estão mais vulneráveis a episódios comportamentais violentos, tanto por parte de quem é cuidado, como por quem cuida.

$\mathrm{Na}$ abordagem intrapessoal, focada no desenvolvimento individual, destaca-se a teoria da aprendizagem social desenvolvida por Bandura (1973). Sob a nomenclatura de teoria do ciclo da violência familiar ou da transmissão transgeracional, esta perspetiva parte do pressuposto de que a "violência é um comportamento aprendido ao longo do ciclo de vida e transmitido entre as diferentes gerações" (Burnight e Mosqueda, 2011: 9). Os indivíduos que, durante a infância, foram sujeitos ou que observaram comportamentos violentos por parte dos pais ou de outros modelos de referência tendem a integrar a violência no seu reportório comportamental e, deste modo, têm maior probabilidade de se comportarem de forma violenta. Pillemer (2005) refere que esta variável não apresenta ainda um nível suficiente de evidência enquanto fator de risco, mas, dada a importância deste aspeto noutras formas de violência interpessoal, deverá continuar a ser investigada.

Da abordagem sociocultural destaca-se a teoria de poder e controlo, desenvolvida inicialmente no âmbito da violência conjugal e enquadrada na perspetiva feminista. A violência conjugal emerge de uma relação de poder desigual e opressiva entre homens e mulheres (Burnight e Mosqueda, 2011). Na população idosa, tal derivaria do idadismo, enquanto discriminação com base na idade, que explicita a tendência para desvalorizar competências e capacidades das pessoas mais velhas. A violência não resulta assim de um aumento das necessidades da vítima, mas do desvio e dependência dos abusadores (Burnight e Mosqueda, 2011; Pillemer e Finkelhor, 1988) que utilizam estratégias coercivas para manter o controlo e o poder na relação.

Das abordagens multissistémicas, salienta-se a teoria ecológica (Bronfenbrenner, 1979), adotada por investigadores da violência conjugal (Burnight e Mosqueda, 2011). O modelo sócio-ecológico deriva da teoria ecológica aliada à perspetiva do ciclo de vida, que enfatiza a importância da variável temporal no contexto da vida familiar. O processo de desenvolvimento humano ao longo do ciclo de vida é condicionado pelas mudanças que configuram os contextos sociais e culturais onde os indivíduos se inserem e estabelecem relações sociais. Procura-se explicar o fenómeno por uma multiplicidade de causas, agrupadas em subsistemas: o macro (sistema de crenças e atitudes sociais), o exo e o meso (sistema de relações de trabalho, educação, família, entre outros) e o microssistema (relações entre pais e filhos).

Este modelo utiliza uma visão multidimensional da violência interpessoal, como resultante da interação entre o indivíduo, suas relações interpessoais, comunidade e traços societais. Combina diversos fatores de risco a partir das diferentes abordagens sobre o problema e agrega vários contributos teóricos (Burnight e Mosqueda, 2011). Schiamberg e Gans (1999) propõem uma 
matriz conceptual para descrever os fatores de risco, distribuídos em três níveis distintos.

O nivel micro contempla as características individuais que podem potenciar o risco de um indivíduo se tornar vítima ou agressor e o modelo tem subjacente uma relação de reciprocidade entre um e outro. No agressor destacam-se os fatores de risco de ordem psicológica (traços de personalidade, problemas de saúde mental e problemas aditivos) e de ordem social (stress derivado dos cuidados, sentimento de sobrecarga e falta de apoio social). Como fatores de risco da vítima identificam-se o sexo, a idade, o estado civil, a situação de incapacidade, o comportamento agressivo e o isolamento social. O nível micro é influenciado, por sua vez, pelas condições sociais (nível meso), incluindo a composição do agregado doméstico (coabitação), os laços e redes sociais (vizinhança, amizade), os fatores socioeconómicos (desemprego, instabilidade laboral, recursos financeiros) e os apoios sociais existentes na comunidade (serviços de apoio social) (Schiamberg e Gans, 1999). Estes fatores funcionarão como aceleradores ou inibidores sociais da vulnerabilidade à violência.

$\mathrm{O}$ nivel macro refere-se aos determinantes estruturais, incluindo as normas culturais e atitudes sociais (que englobam o idadismo, o sexismo e as atitudes face à violência), assim como as políticas sociais públicas.

O modelo sócio-ecológico não é determinístico, nem causal. Os fatores de risco são tidos na sua interação conjunta entre os três níveis descritos; ou seja, os determinantes individuais da vítima e do agressor constituem fatores de risco que são moldados pela estrutura social. As condições sociais podem aumentar o risco de violência, e os traços societais, como o quadro jurídico-legal de proteção à pessoa idosa ou as políticas de família e velhice, podem condicionar os cenários sociais onde a violência ocorre, prevenindo ou respondendo aos determinantes individuais e sociais.

Os fatores de risco mais investigados na literatura sobre a prevalência do fenómeno inserem-se, maioritariamente, nos níveis micro e meso e incluem variáveis respeitantes quer à vítima, quer ao agressor, embora a conceptualização incida, usualmente, numa perspetiva unidirecional, reduzida à relação de dependência entre vítima e agressor.

Nos estudos de prevalência de base populacional que estimaram o risco relativo de diferentes fatores, a explicitação dos modelos teórico-conceptuais de partida é quase sempre omissa. No entanto, a identificação dos fatores de risco, ou seja, das variáveis que incrementam ou diminuem a probabilidade de ocorrência da violência decorre das perspetivas teóricas analisadas. O foco de explicação baseia-se nas características individuais da vítima (teorias intrapessoais), na natureza da relação entre vítima e agressor (teorias interpessoais), nas normas e valores culturais e sociais (teorias socioculturais) ou nas interações e impacto dos diferentes sistemas (exo e meso) nos determinantes individuais da vítima e do agressor (teoria multissistémica). 


\subsection{Fatores de risco de violência contra as pessoas idosas}

A investigação nas últimas décadas tem indicado um conjunto diversificado de fatores de risco (Pillemer, 2005) ou de determinantes que podem assumir um carácter potenciador ou protetor da violência.

No contexto dos estudos epidemiológicos, a identificação de fatores de risco associados à ocorrência de determinado evento pode ser realizada através de estudos transversais ou longitudinais. A maioria dos estudos revistos no presente artigo (Quadro 1) são estudos epidemiológicos transversais, com exceção do trabalho de Lachs e colaboradores (1997). Tratou-se do único estudo prospetivo, que se baseou numa coorte de pessoas com $65+$ anos seguidas durante nove anos.

A identificação dos fatores de risco nem sempre foi explicitamente mencionada nos estudos, cuja principal finalidade foi a estimação da prevalência da violência. Consequentemente, os potenciais fatores de risco identificados em alguns dos estudos (Grande et al., 2000; Marmolejo, 2008; Naughton et al., 2012) resultam da análise de frequências aplicada às características de vítimas e agressores e a aspetos contextuais e/ou socioculturais. Dado que a análise de frequências só disponibiliza informação descritiva, é também usual o desenvolvimento de testes estatísticos para comparação de médias ou de proporções da característica observada em vítimas e não vítimas.

Esta abordagem apenas permite evidenciar as características ou situações mais frequentes na amostra ou em categorias (tais como vítimas e não vítimas), mas não identifica fatores presentes antes da ocorrência do fenómeno. Pillemer (2005) refere serem escassos os estudos que utilizam o método de estudo caso-controlo, que permite comparar a frequência das variáveis que são potenciais fatores de risco em dois grupos: casos (vítimas) e controlos (não vítimas).

Para identificar fatores de risco, ou seja, para avaliar se determinadas características são determinantes da violência, é necessário comprovar a existência de uma associação entre a ocorrência de violência e as características individuais e os aspetos contextuais e/ou socioculturais de interesse, com controlo de aspetos adicionais que podem confundir e/ou modificar tal associação. Neste sentido, é usual o recurso à análise de regressão logística multivariada, que avalia a significância da associação entre a probabilidade de ocorrência de violência e fatores de risco, estimando o respetivo efeito protetor/potenciador. Somente cinco dos estudos revistos (Acierno et al.,2010; Lachs et al., 1997; Laumann, Leitsch e Waite, 2008; Lowenstein, Eisikovits e Band-WWinterstein, 2009; O'Keefe et al., 2007) aplicaram a regressão logística na identificação de fatores de risco e na quantificação do risco relativo. Porque resultantes da comprovação de associações estatisticamente significativas, os fatores de risco evidenciados pelos cinco estudos citados (Quadro 1) devem ser valorizados face aos descritos pelos restantes, que serão fatores potencialmente influentes. 


\subsubsection{Potenciais fatores de risco das vítimas}

A nível micro, enquanto fatores de risco da vítima, têm sido analisadas características sociodemográficas (género, idade, estado civil), determinantes em saúde (autoperceção do estado de saúde, doenças crónicas, capacidade funcional, depressão, capacidade cognitiva, entre outros) e aspetos socioeconómicos (rendimento, situação perante o trabalho, habilitações literárias e utilização de serviços de âmbito social), sintetizados no Quadro 1 (páginas seguintes).

Relativamente às características sociodemográficas, a vítima-tipo foi inicialmente descrita como uma mulher de 75 ou mais anos (Wolf, 1992). De facto, metade dos estudos reportou que as mulheres estavam potencialmente em maior risco de serem vítimas de violência global (Marmolejo, 2008) ou de tipos específicos de violência (Laumann, Leitsch e Waite, 2008; O'Keefe et al., 2007; Podnieks, 1993). Contudo, os dados não permitem falar do género enquanto fator de risco consistente. A desproporção demográfica entre homens e mulheres, particularmente nos grupos etários mais envelhecidos pela sobremortalidade masculina, pode constituir um argumento explicativo (Crichton, 1999). Além disso, muitas vezes os estudos não diferenciam o contexto e o tipo de violência, o que poderá também explicar a variabilidade dos resultados.

$\mathrm{O}$ estado civil parece estar associado ao género, sobretudo na violência conjugal. Alguns investigadores (Laumann, Leitsch e Waite, 2008; Lowenstein, Eisikovits e Band-WWinterstein, 2009) verificaram que a violência física e sexual foi sobretudo cometida pelos parceiros, tendo os casados, em comparação com os não casados, evidenciado um risco acrescido. Alguns autores sustentam que género e estado civil são fatores de risco da violência conjugal, a qual não é distinguida nos diferentes estudos da violência exercida em idades mais avançadas (Desmarais, Reeves e Gray, 2007). Os contornos da violência conjugal fazem parte de uma história de vida de agressões, muito pouco associada à questão da idade (Gil, 2010). As dinâmicas da relação estarão presentes desde a formação da violência conjugal, pelo que alguns dos seus fatores de risco se encontram ao longo do ciclo vital e poderão, em idades mais avançadas, aumentar o risco de violência (Desmarais, Reeves e Gray, 2007). Nos estudos revistos, o estado civil parece estar mais consistentemente associado à violência financeira, sendo as pessoas idosas que vivem sozinhas (solteiras, separadas ou divorciadas) mais vulneráveis (Acierno et al., 2010; Podnieks, 1993; O’Keefe et al., 2007).

A idade da vítima poderá não ser em si um fator de risco, mas pode assumir um papel relevante quando associada à deterioração do estado de saúde e aumento da incapacidade funcional. Dois estudos concluem que a idade mais avançada $(85+$ anos) é um potencial fator de risco da violência psicológica, financeira ou de negligência enquanto noutros dois estudos os grupos etários mais jovens apresentavam maior vulnerabilidade à violência psicológica, física e sexual (Acierno et al., 2010; Podnieks, 1993). 


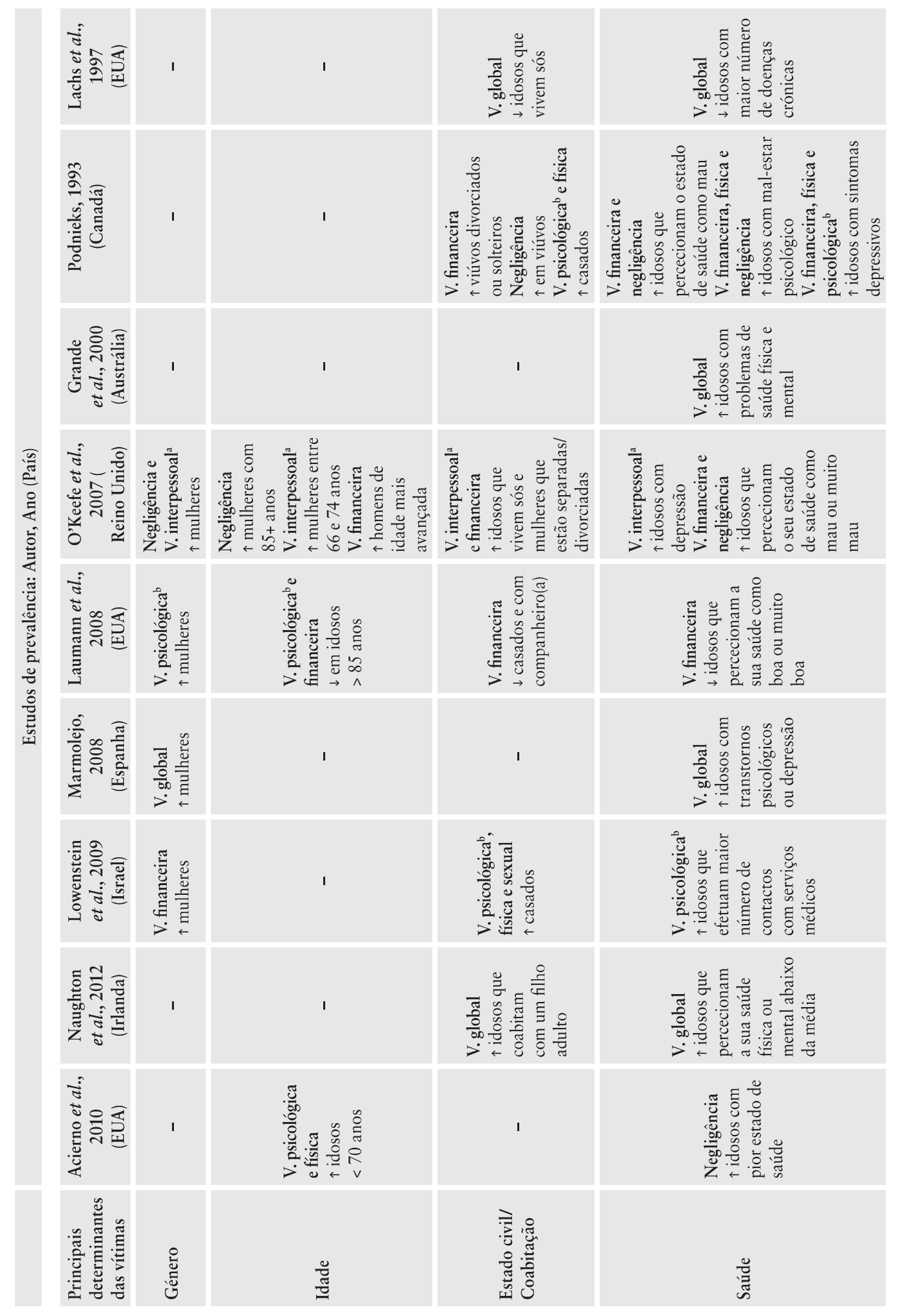




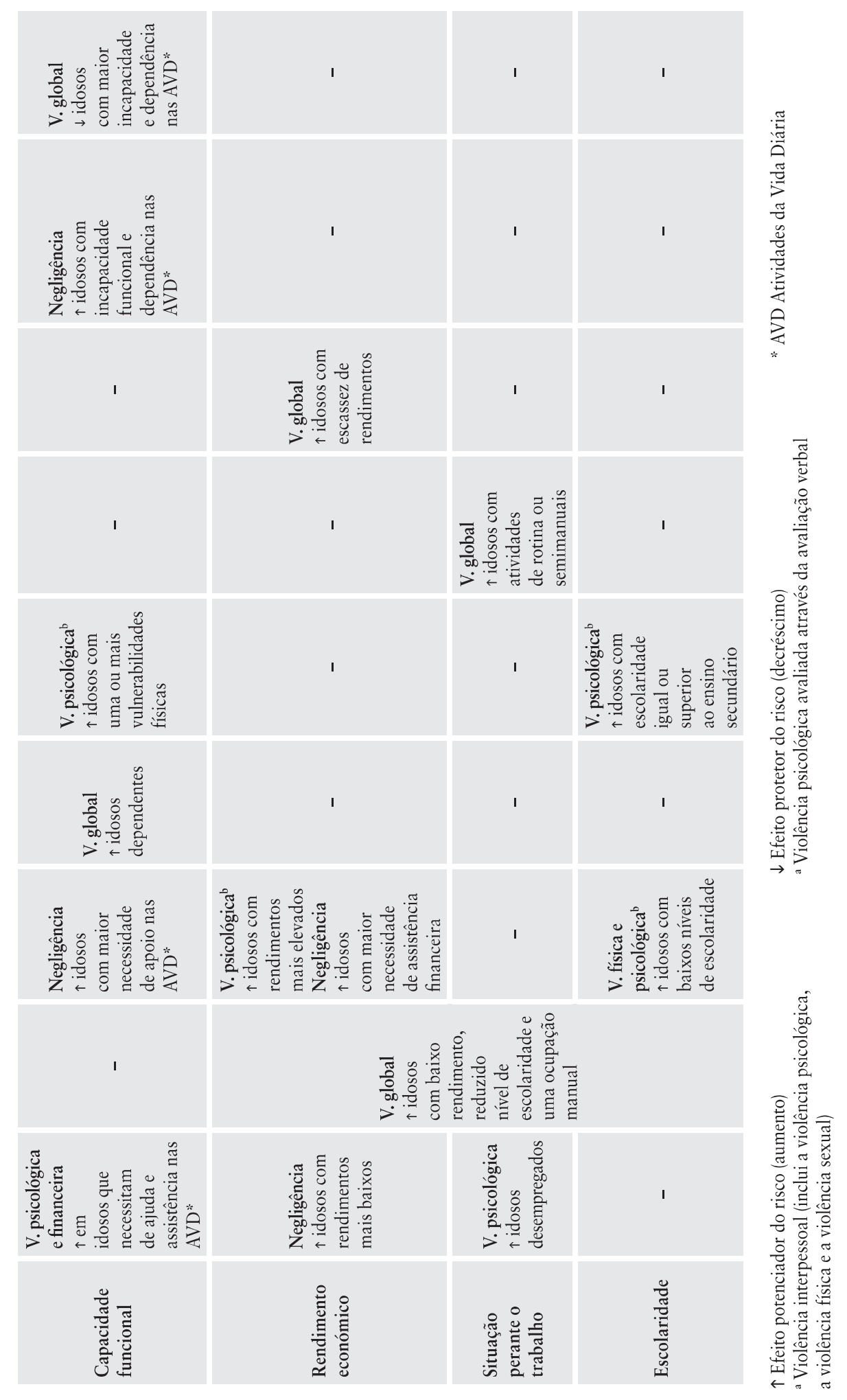




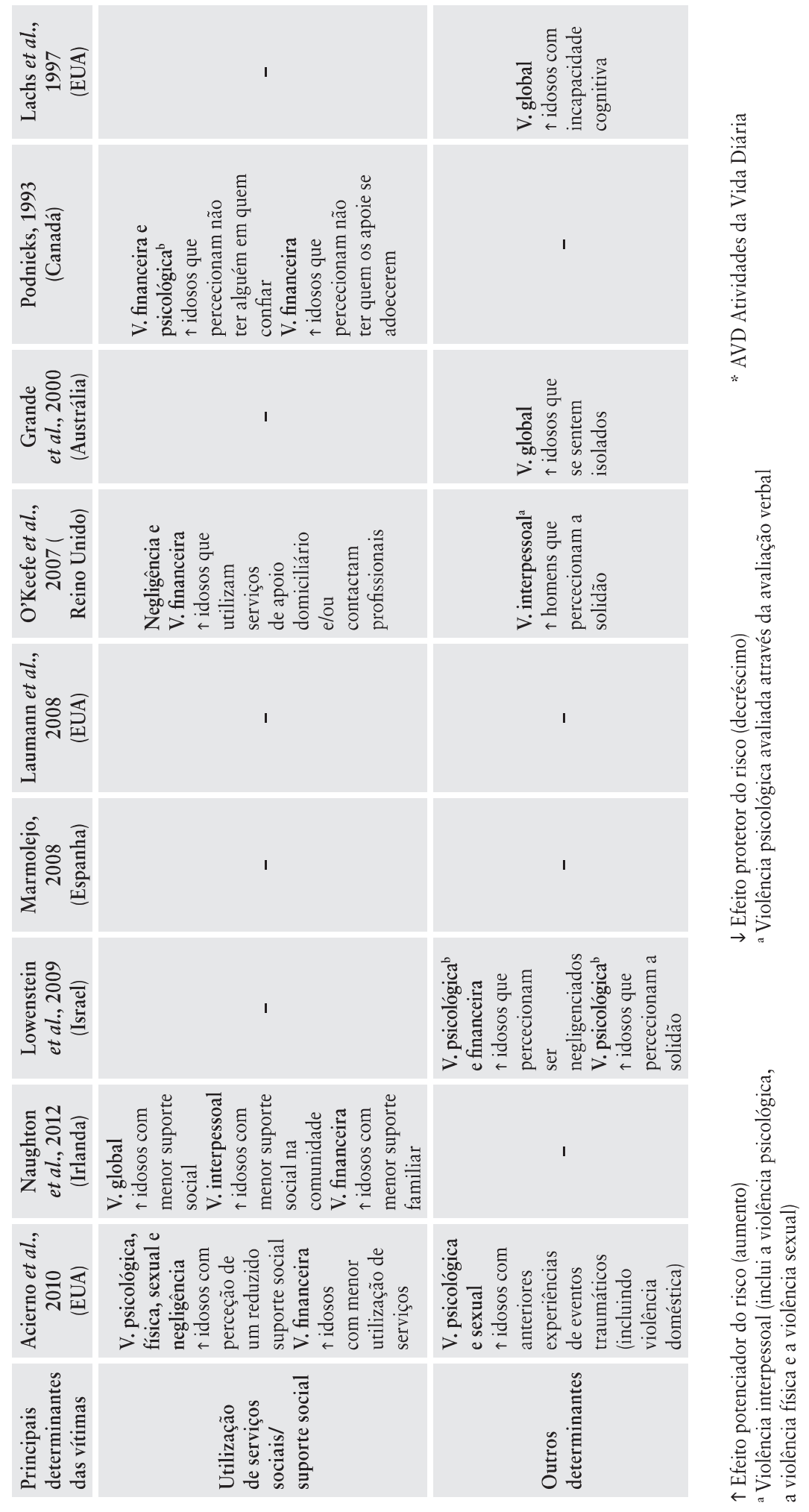


Os determinantes de saúde da vítima são amplamente estudados e englobam um conjunto heterogéneo de variáveis que incluem a autoperceção do estado de saúde, a capacidade funcional (usualmente avaliada através das Atividades de Vida Diária - AVD), a morbilidade e o número de contactos com serviços de saúde.

A negligência surge como o tipo de violência que de forma mais consistente se associa a estes determinantes. Um pior (mau ou muito mau) estado de saúde (Acierno et al., 2010; Podnieks, 1993; O'Keefe et al., 2007) e um grau de incapacidade funcional mais elevado nas AVD (Lowenstein, Eisikovits e BandWWinterstein, 2009; Podnieks, 1993) foram identificados como potenciais fatores de risco para a negligência. No entanto, não é evidente a relação entre o estado de saúde e a incapacidade funcional: constituirá o estado de saúde isoladamente um fator de risco, ou será o aumento da incapacidade funcional consequência de um pior estado de saúde, que poderá aumentar o risco face à violência?

O único estudo que analisou esta relação (Lachs et al., 1997) apresentou resultados contraditórios. A incapacidade funcional nas AVD aumenta a possibilidade de se ser vítima de violência em geral, mas o maior número de doenças crónicas constitui um fator protetor daquele risco; talvez pelo maior número de contactos com profissionais.

No estudo de Acierno e colaboradores (2010), a autoperceção negativa do estado de saúde foi um fator duplicador do risco de negligência. Já na violência psicológica e financeira, esta variável só constituía um fator de risco quando associada à incapacidade funcional e, consequentemente, às maiores necessidades de apoio nas AVD. A causalidade reversa surge aqui como uma via de explicação da associação entre estas dimensões, apenas acessível a estudos epidemiológicos com desenhos longitudinais prospetivos.

Em suma, não é evidente o papel que estado de saúde e grau de incapacidade funcional terão, isoladamente ou em associação, na explicação da maior vulnerabilidade à violência.

A saúde mental tem sido igualmente abordada como fator de risco, contemplando a avaliação da capacidade cognitiva e da depressão ou sintomatologia depressiva. Alguns estudos, ao avaliar a existência de patologia mental, observaram uma maior percentagem de vítimas de violência com problemas de saúde mental (Grande et al., 2000).

Os sintomas depressivos, enquanto fator de risco, foram analisados em três estudos. No Reino Unido (O'Keefe et al., 2007), a sintomatologia depressiva foi entendida como um fator precipitante da violência em geral, enquanto no Canadá (Podnieks, 1993) esta variável aumentou a vulnerabilidade à violência financeira, física e psicológica. No único estudo prospetivo revisto (Lachs et al., 1997), esta variável não demostrou ser significativa na explicação da ocorrência de violência. A sintomatologia depressiva, embora pareça ser relevante, e deva ser explorada, é de difícil valorização como fator de risco, pois 
não é possível concluir se os sintomas depressivos constituem a causa ou são a consequência do próprio processo de vitimação.

O fenómeno da violência também parece estar associado à demência. No entanto, nos estudos analisados, a incapacidade cognitiva não é avaliada através de um instrumento estandardizado e, quando considerada, a demência constitui, por vezes, um critério de exclusão (Acierno et al., 2010; Laumann, Leitsch e Waite, 2008; Marmolejo, 2008; Podnieks, 1993). A não avaliação da capacidade cognitiva pode constituir uma limitação dos estudos, ainda que a própria natureza e a extensão dos questionários excluam pessoas com défices cognitivos moderados ou severos. Dos dois estudos (Lachs et al., 1997; Laumann, Leitsch e Waite, 2008) que avaliaram o défice cognitivo, apenas um apresentou resultados significativos. Tratou-se do único estudo prospetivo (Lachs et al., 1997) que concluiu que as pessoas com incapacidade cognitiva apresentavam três vezes mais probabilidade de virem a ser vítimas de violência.

Quando a população-alvo inclui pessoas idosas com diagnóstico de demência, esta variável tem sido associada ao aumento do risco de violência (Lachs et al., 1997), particularmente quando as vítimas sofrem de Alzheimer, doença descrita como fator de risco na violência física (Lachs et al., 1997).

Os determinantes socioeconómicos que mais frequentemente integram inquéritos de prevalência referem-se à situação económica, à condição perante o trabalho e às habilitações literárias. A informação apresentada no Quadro 1 permite concluir que os rendimentos mais baixos e as necessidades económicas são potenciadores da negligência (Acierno et al., 2010; Lowenstein, Eisikovits e Band-WWinterstein, 2009), enquanto os rendimentos mais elevados surgem mais associados à violência psicológica (Lowenstein, Eisikovits e BandWWinterstein, 2009).

Quanto à escolaridade, os estudos apresentam resultados contraditórios (Acierno et al., 2010; Lowenstein, Eisikovits e Band-WWinterstein, 2009). No estudo irlandês (Naughton et al., 2012) foi identificada uma associação entre a violência e a classe social resultante da agregação do rendimento, habilitações literárias e situação profissional. Este estudo concluiu que pessoas oriundas de profissões operárias, com parcos rendimentos e baixa escolaridade, tinham maior possibilidade de serem vítimas de violência.

\subsubsection{Potenciais fatores de risco dos agressores}

A nível micro e de acordo com o modelo sócio-ecológico, as características do agressor tendem a ser mais relevantes para a ocorrência da violência do que as da vítima (Marmolejo, 2008). Apesar de contemplarem variáveis sociodemográficas, os fatores de risco do agressor relacionam-se mais com traços de personalidade, problemas de saúde mental, stress do cuidador, competências de coping e exposição à violência na infância ou adolescência (Quadro 2, v. pp. 90-91). 
Os agressores são maioritariamente familiares diretos, particularmente parceiros e filhos. No Canadá (Podnieks, 1993), Reino Unido (O'Keefe et al., 2007) e Austrália (Grande et al., 2010), identificaram-se os parceiros como os principais responsáveis pela violência. Em Espanha (Marmolejo, 2008), quando os cuidadores foram questionados, os filhos constituíam a maioria dos agressores. Os idosos irlandeses (Naughton et al., 2012) também identificaram, como principais agressores, os filhos, seguidos de outros familiares e parceiros.

A violência física e sexual foi sobretudo exercida pelos parceiros do sexo masculino (Lowenstein, Eisikovits e Band-WWinterstein, 2009; O'Keefe et al., 2007), enquanto a violência financeira foi, maioritariamente, cometida pelos filhos e outros familiares (Naughton et al., 2012; Laumann, Leitsch e Waite, 2008). Vários estudos têm também reportado que, ainda que com menor prevalência, os amigos, vizinhos, conhecidos (não familiares) e cuidadores formais também são agressores (Marmolejo, 2008; O’Keefe et al., 2007; Podnieks, 1993).

O género e a idade do agressor parecem não ser determinantes inequívocos. Nos estudos em que os agressores eram sobretudo os parceiros, as mulheres reportaram mais frequentemente terem sido vítimas, exceto na violência financeira e negligência, motivo pelo qual os homens com mais de 64 anos predominavam no grupo dos agressores (Grande et al., 2010; Marmolejo, 2008; Podnieks, 1993).

No estudo irlandês (Naughton et al., 2012) os filhos, com idades compreendidas entre 31 e 64 anos, foram identificados como os principais agressores, independentemente do género.

Em Espanha, quando se questionaram as pessoas idosas (Marmolejo, 2008), os agressores indicados eram maioritariamente parceiros, sendo quase metade do género masculino. Mas, no inquérito dirigido aos cuidadores (Marmolejo, 2008), a maioria dos agressores eram as filhas (82\% dos cuidadores inquiridos eram mulheres). Em suma, provavelmente, o género e a idade são características associadas a outras variáveis, como a relação do agressor com a vítima e o tipo de violência.

Os determinantes sociossituacionais do agressor, mais concretamente a situação perante o trabalho e a situação económica, foram avaliados em seis países. No Reino Unido (O’Keefe et al., 2007), 2\% dos agressores estavam desempregados, enquanto na Irlanda (Naughton et al., 2012) se verificou o mesmo em 50\% dos agressores. Em Israel (Lowenstein, Eisikovits e BandWWinterstein, 2009), com exceção da negligência, quando os agressores eram os filhos, um elevado número encontrava-se desempregado. Na Austrália (Grande et al., 2010), 8,8\% dos agressores tinham dificuldades económicas, enquanto no Canadá (Podnieks, 1993) se observou que a dependência financeira do agressor face à pessoa idosa fomentava a ocorrência de violência física. 


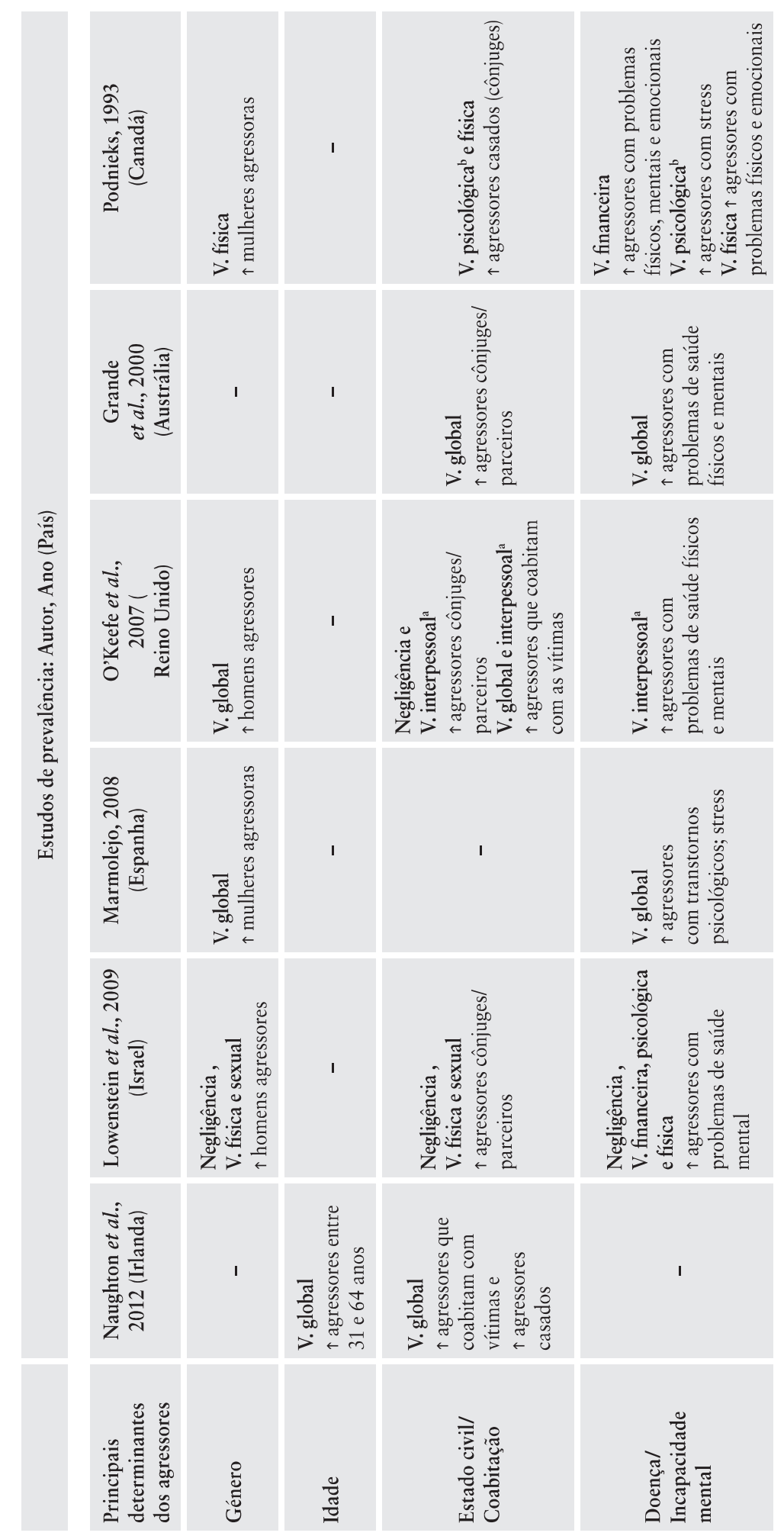



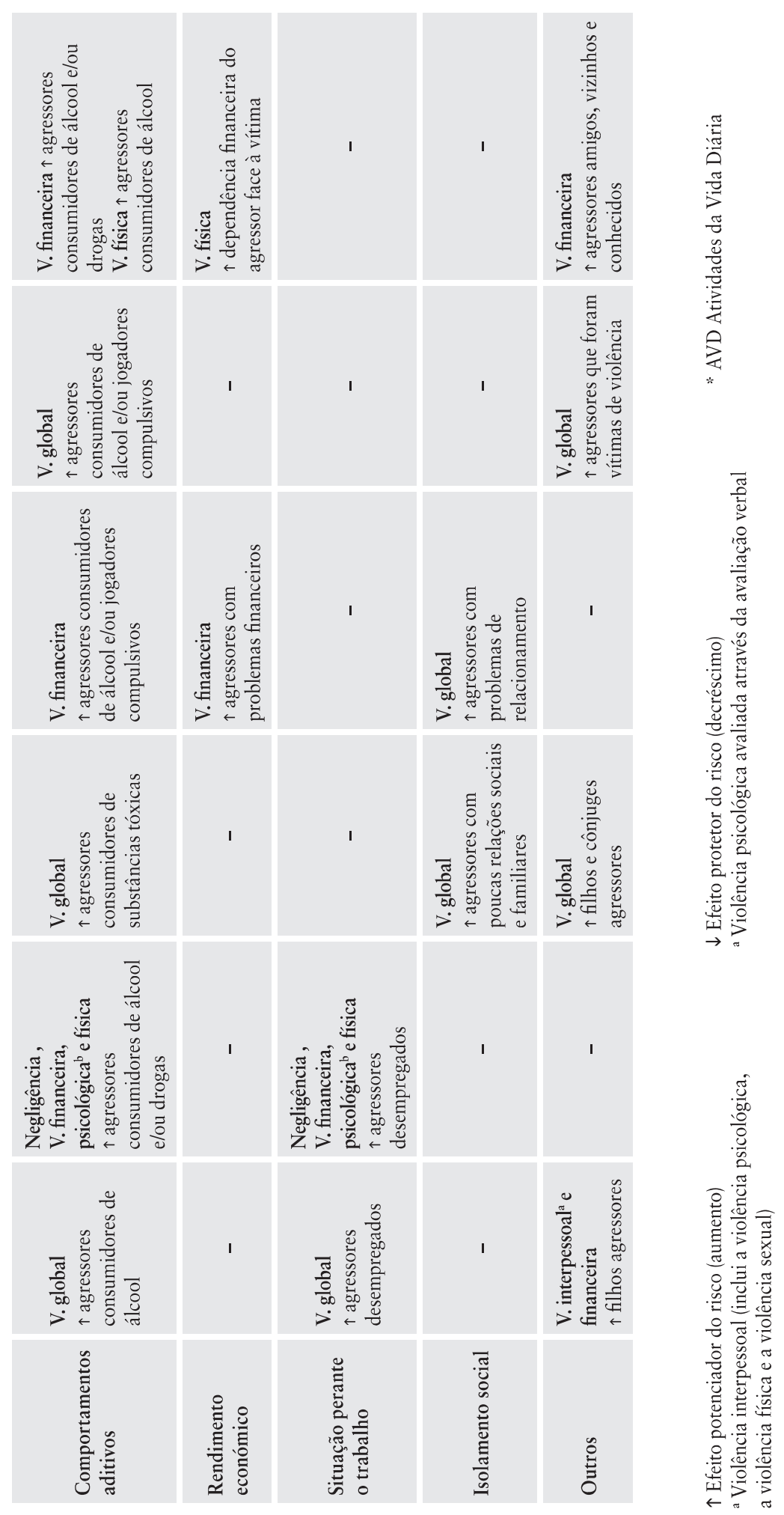
Em Espanha (Marmolejo, 2008), 47,4\% das pessoas idosas relataram que o seu rendimento era a única fonte de receita do agregado.

Os problemas de saúde mental do agressor, o abuso do álcool e de drogas e os problemas associados ao jogo constituem importantes fatores de risco, igualmente identificados pelos diferentes estudos (Grande et al., 2010; Lowenstein, Eisikovits e Band-WWinterstein, 2009; Marmolejo, 2008; Naughton et al., 2012; O'Keefe et al., 2007; Podnieks, 1993). Menos consensuais são os problemas comportamentais ao nível das relações interpessoais e/ou isolamento social, apesar de evidências em alguns estudos (Marmolejo, 2008; Podnieks, 1993).

No que concerne a exposição do agressor à violência na sua infância ou adolescência, como vítima ou mera testemunha, esta variável não foi avaliada por nenhum dos estudos revistos (Pillemer, 2005).

\subsubsection{Potenciais fatores de risco contextuais}

A coabitação é um dos fatores de risco mais referidos na literatura (Lachs et al., 1997; Pillemer, 2005; Wolf, 1992); contudo, dos estudos revistos, apenas um (O'Keefe et al., 2007) indicou a coabitação com descendentes como fator de risco putativo.

A rede social de suporte tem sido associada à ocorrência da violência, muitas vezes entrelaçada com o conceito de isolamento social, enquanto sistema de relações sociais que o indivíduo estabelece na esfera social, desempenhando duas funções: de sociabilidade e de suporte social. O suporte social "inscreve-se num sistema mais amplo das relações sociais, incluindo um número de atividades que podem ir, desde o apoio emocional, instrumental, económico" (Gil, 2010: 310). Podem distinguir-se duas fontes de suporte social: a informal, que inclui indivíduos das relações familiares, de amizade e de vizinhança, e a formal, que abrange os serviços de saúde ou de âmbito social.

Alguns estudos têm evidenciado que o isolamento social da pessoa idosa potencia a vitimação, nomeadamente quando a rede social de suporte é frágil ou inexistente (Pillemer, 2005). Os estudos revistos indicam que a ausência de uma rede de suporte constitui um potencial fator de risco, em geral ou na violência psicológica, financeira e negligência.

No caso irlandês (Naughton et al., 2012), o suporte social foi definido como o número de pessoas disponíveis para prestar apoio emocional, instrumental e pessoal. As pessoas idosas com uma rede de suporte diminuta tinham três a quatro vezes mais possibilidades de serem vítimas. Também Lachs e colaboradores (1997) confirmaram que um apoio social diminuto incrementava significativamente o risco de vitimação, enquanto Acierno e colaboradores (2010) observaram que o suporte social fraco quadruplicava o risco de negligência. 


\section{Considerações finais}

Ao longo das últimas décadas, a investigação sobre a prevalência da violência na população idosa tem originado uma vasta e heterogénea evidência ao nível dos seus fatores de risco.

A revisão efetuada fundamentou-se em resultados de estudos de prevalência de base populacional. Os estudos diferem entre si em múltiplos aspetos: conceitos operatórios; desenhos de estudo e métodos estatísticos utilizados. O pequeno número de casos de violência identificados e os diferentes métodos de análise usados na identificação dos fatores mais associados ao fenómeno requerem investigação adicional dos fatores de risco, designadamente da sua sustentabilidade do ponto de vista epidemiológico e estatístico. Como refere Pillemer (2005), é necessária uma leitura crítica destas variáveis, uma vez que a investigação desenvolvida também se caracteriza: por uma definição pouco clara do objeto, nem sempre diferenciada por tipos de violência; pela utilização de diferentes critérios de determinação da população em risco; pelo emprego de diferentes desenhos de estudo, onde escasseiam os estudos de caso-controle e os estudos prospetivos; e pela utilização de diferentes métodos de mensuração dos fatores de risco.

Face a este panorama, Lachs e Pillemer (2004) propõem a diferenciação dos fatores de risco de acordo com a evidência empírica, agrupando-os em duas grandes categorias: plausíveis e consubstanciados. Estes últimos apresentam consistência empírica em termos de investigação. Alguns determinantes da vítima, do agressor e do contexto familiar parecem ser fatores plausíveis de potenciar o risco. A incapacidade funcional, a demência e a depressão, bem como o contexto socioeconómico, constituem fatores plausíveis, que necessitam de maior investigação. No que respeita à vítima, o sexo, a idade, o estado de saúde e o isolamento social surgem, frequentemente, como fatores de risco já consubstanciados.

No caso do agressor, os problemas de saúde mental, a dependência económica e a coabitação com a vítima são também fatores de risco consubstanciados, enquanto os plausíveis abrangem os problemas de relacionamento interpessoal, o stress do cuidador e a transmissão geracional de comportamentos violentos.

Como se concluiu dos estudos revistos, a ocorrência da violência varia de acordo com o tipo de agressão, as características da vítima e o tipo de relação desta com o agressor, bem como com variáveis contextuais. Estes resultados remetem-nos para o quadro teórico que alia, de forma mais integrada, as condições de ocorrência da violência e os fatores associados, ou seja, o modelo sócio-ecológico, quase sempre omisso nos estudos analisados.

Relativamente à vítima, as características sociodemográficas, como o sexo, a idade, a escolaridade e o estado de saúde (a doença e a incapacidade), são variáveis que influenciam consensualmente a ocorrência da violência. Em contrapartida, os traços do agressor variam de acordo com o tipo de violência 
e, apesar de os estudos revistos identificarem problemáticas associadas aos agressores (problemas de saúde mental, de consumos, dependência económica, problemas de relacionamento interpessoal), estes aspetos necessitam de investigação adicional.

A revisão efetuada revelou a necessidade de investigação adicional sobre os fatores de risco e de proteção e a insuficiente explicitação dos modelos teóricos conceptuais. A coerência entre fatores de risco e as perspetivas teóricas sobre a violência nem sempre surge de forma articulada e carece de relevância interpretativa das estimativas obtidas.

Atendendo a que os nove estudos de prevalência revistos utilizaram diferentes critérios de determinação da população em risco e distintas abordagens metodológicas na identificação dos fatores associados a tipos de violência, nem sempre concordantes, a maior limitação do presente estudo reside na comparabilidade dos resultados.

Procurou-se identificar os principais fatores que potenciam ou reduzem o risco de vitimação das pessoas idosas, em contexto familiar, na convicção de que uma compreensão mais apurada da interação entre aqueles fatores contribui para a reflexão sobre as formas mais eficazes para promover a prevenção e o combate deste problema.

\section{Bibliografia}

ACIERNO, R. et al. (2010), "Prevalence and correlates of emotional, physical, sexual, and financial abuse and potential neglect in the United States: the national elder mistreatment study". American Journal of Public Health, 2: 292-297.

ATTIAS-DONFUT, C. (directeur) (1995), Les Solidarités entre générations. Vieillesse, familles, État. Paris: Nathan.

BENGSTON, V. L. (2001), "Beyond the nuclear family: the increasing importance of multigenerational bonds". Journal of Marriage and the Family, 63: 1-16.

BRONFENBRENNER, U. (1979), The Ecology of Human Development: Experiments by nature and design. Cambridge, MA: Harvard University Press.

BURNIGHT, K.; MOSQUEDA, L. (2011) Theoretical Model Development in Elder Mistreatment. California: University of California, Irvine, School of Medicine, Program in Geriatrics.

CRICHTON, S. J. et al. (1999), "Elder abuse: feminist and ageist perspectives”, Journal of Elder Abuse \& Neglect, 10(3-4): 115-130.

COENEN-HUTHER, J.; KELlERHALS, J.; ALLMEN, M. (1994), Les Réseaux de solidarité dans la famille. Suisse: Réalités Sociales.

DESCMARAIS, S. L.; REEVES, K. A. (2007), "Gray, black, \& blue: The state of research and intervention for intimate partner abuse among elders". Behavioral Sciences of the Law, 25: 377-391.

FERNANDES, A. A. (1997), Velhice e Sociedade: Demografia, familia e politicas sociais em Portugal. Oeiras: Celta Editora.

FERREIRA-ALVES J. (2005), “Abuso e negligência de pessoas idosas: contributos para a sistematização de uma visão forense de maus-tratos”, in R. A. Gonçalves e C. Machado (editores), Psicologia Forense. Coimbra: Quarteto Editora. 
GIL, A. P. M. (2010), Heróis do Quotidiano: dinâmicas familiares na dependência. Lisboa: Textos Universitários de Ciências Sociais e Humanas, Fundação Calouste Gulbenkian e Fundação para a Ciência e a Tecnologia, Ministério da Ciência, Tecnologia e Ensino Superior.

GRANDE, E. D. et al. (2000), Interpersonal Violence and Abuse Survey. South Australia: South Australian Department of Human Services.

LACHS, M. S. et al. (1997), "Risk factors for reported elder abuse and neglect: a nine-year observational cohort study", The Gerontologist, 37(4): 469-474.

LACHS, M. S. \& PILLEMER, K. (2004), “Elder abuse”. Lancet, 364(2): 1263-1272.

LAUMANN, E. O.; LEITSCH, S.A. \& WAITE, L. J. (2008), "Elder mistreatment in the United States: prevalence estimates from a nationally representative study", Journals of Gerontology: Social Sciences, 63(4): 248-254.

LAVOIE, J. P. (2000), Familles et soutien aux parents âgés dépendants. Paris: L'Harmattan.

LOWENSTEIN, A. et al. (2009), "Is elder abuse and neglect a social phenomenon? Data from the first national prevalence survey in Israel”, Journal of Elder Abuse and Neglect, 21: 253-277.

LUESCHNER, K. \& PILLEMER, K. (1998), "Intergenerational ambivalence: A new approach to the study of parent-child relations in later life", Journal of Marriage and the Family, 60: 413-445.

MARMOLEJO, I. M. (2008), Maltrato de personas mayores en la família en España. Valencia: Fundación de la Comunitat Valenciana para el estúdio de la Violencia.

NAUGHTON, C.; DRENNAN, J.; LYONS, I.; LAFFERTY, A.; TREACY, M. P.; PHELAN, A.; O'LOUGHLIN, A. \& DELANEY, L. (2012), "Elder abuse and neglect in Ireland: Results from a national prevalence survey". Age and Ageing, 41(1): 98-103. doi: 10.1093/ageing/ afr107.

O'KEEFE, M. et al. (2007), UK Study of Abuse and Neglect of Older People: Prevalence survey report. London: National Centre for Social Research and King's College.

PAVEZA, G. P. et al. (1992), "Severe family violence and Alzheimer's disease: prevalence and risk factors", The Gerontologist, 32(4): 493-497.

PEREL-LEVIN, S. (2008), Discussing Screening for Elder Abuse at Primary Health Care Level. Geneva: World Health Organization Press.

PILLEMER, K. \& FINKELHOR, D. (1988), “The prevalence of elder abuse: a random sample survey", The Gerontologist, 28: 51-57.

PILLEMER, K. (2005) "Factores de riesgo del maltrato de mayores", in Marmolejo II (editora), Violencia contra personas mayores. Barcelona: Coleccíon Estudios sobre Violencia, 18-28.

PODNIEKS, E. (1993), "National survey on abuse of the elderly in Canada", Journal of Elder Abuse and Neglect, 4: 55-58.

SCHIAMBERG, L. B. \& GANS, D. (1999), "An ecological framework for contextual risk factors in elder abuse by adult children", Journal of Elder Abuse and Neglect, 1(1): 79-103.

SEVER, A. (2009), "More than wife abuse that has gone old: a conceptual model for violence against the aged in Canada and the US", Journal of Comparative Family Studies, 22: 279-292.

WOLF, R. S. (1992), "Victimization of the elderly: elder abuse and neglect", Clinical Gerontology, 2: $269-272$.

WORLD HEALTH ORGANISATION (OMS)/ International Network for the Prevention of Elder Abuse (INPEA) (2002), Missing Voices: Views of older persons on elder abuse. Geneva: World Health Organization.

TORTOSA, J. M. (2004), Personas Mayores y Malos Tratos. Madrid: Psicología Pirámide.

ZARIT, S. H.; REEVER, K. E. \& BACH-PETERSON, J. (1980), "Relatives of the impaired elderly: correlates of feelings of burden", The Gerontologist, 20(6): 649-655. 